\title{
Evaluation of Metabarcoding Primers for Analysis of Soil Nematode Communities
}

\author{
Md. Maniruzzaman Sikder ${ }^{1,2} \mathbb{D}$, Mette Vestergård ${ }^{1}\left(\mathbb{D}\right.$, Rumakanta Sapkota ${ }^{3} \mathbb{D}$, Tina Kyndt ${ }^{4}(\mathbb{D}$ \\ and Mogens Nicolaisen $1, * \mathbb{D}$ \\ 1 Department of Agroecology, Faculty of Technical Sciences, Aarhus University, 4200 Slagelse, Denmark; \\ mms@agro.au.dk (M.M.S.); mvestergard@agro.au.dk (M.V.) \\ 2 Department of Botany, Faculty of Biological Sciences, Jahangirnagar University, \\ 1342 Savar, Dhaka, Bangladesh \\ 3 Department of Environmental Science, Faculty of Technical Sciences, Aarhus University, \\ 4000 Roskilde, Denmark; rs@envs.au.dk \\ 4 Department of Molecular Biotechnology, Faculty of Bioscience Engineering, Ghent University, \\ 9000 Gent, Belgium; tina.kyndt@ugent.be \\ * Correspondence: mn@agro.au.dk; Tel.: +45-24757668
}

Received: 5 August 2020; Accepted: 7 October 2020; Published: 9 October 2020

\begin{abstract}
While recent advances in next-generation sequencing technologies have accelerated research in microbial ecology, the application of high throughput approaches to study the ecology of nematodes remains unresolved due to several issues, e.g., whether to include an initial nematode extraction step or not, the lack of consensus on the best performing primer combination, and the absence of a curated nematode reference database. The objective of this method development study was to compare different primer sets to identify the most suitable primer set for the metabarcoding of nematodes without initial nematode extraction. We tested four primer sets for amplicon sequencing: JB3/JB5 (mitochondrial, I3-M11 partition of COI gene), SSU_04F/SSU_22R (18S rRNA, V1-V2 regions), and Nemf/18Sr2b (18S rRNA, V6-V8 regions) from earlier studies, as well as MMSF/MMSR (18S rRNA, V4-V5 regions), a newly developed primer set. We used DNA from 22 nematode taxa, 10 mock communities, 20 soil samples, 4 root samples, and one bulk soil. We amplified the target regions from the DNA samples with the four different primer combinations and sequenced the amplicons on an Illumina MiSeq sequencing platform. We found that the Nemf/18Sr2b primer set was superior for detecting soil nematodes compared to the other primer sets based on our sequencing results and on the annotation of our sequence reads at the genus and species ranks. This primer set generated $74 \%$ reads of Nematoda origin in the soil samples. Additionally, this primer set did well with the mock communities, detecting all the included specimens. It also worked better in the root samples than the other primer set that was tested. Therefore, we suggest that the Nemf/18Sr2b primer set could be used to study rhizosphere soil and root associated nematodes, and this can be done without an initial nematode extraction step.
\end{abstract}

Keywords: nematode diversity; soil; rhizosphere; environmental; NGS

\section{Introduction}

Nematodes are highly diverse and abundant metazoans with a worldwide distribution [1,2]. Generally, nematologists have relied on classical morphology-based taxonomy along with biochemical or molecular methods for nematode identification [3,4]. Morphological identification is difficult, requires taxonomic expertise [5], and often becomes challenging when it comes to identifying nematodes at lower taxonomic levels [6]. DNA-based identification has eased the task of taxonomic nematode identification in recent years [7-10]. 
Initially, the barcoding approach was introduced for species-level detection [11-13]. The mitochondrial cytochrome oxidase I gene (COI gene) has been successfully used as barcode for the identification of nematodes and for resolving taxonomic relationships among closely related species [14-16]. The COI gene has been shown to provide a greater taxonomic resolution than the small subunit (18S rRNA) rDNA [17]. The potential of COI gene-based barcoding specific to particular groups of nematodes has been explored on pure DNA samples of various nematode taxa including root-knot nematodes [18], marine nematodes [16], Aphelenchoididae [19] and Pratylenchus [20]. A lack of suitable COI-based consensus primers for the detection of nematode diversity in comparison to 18S-based primers limits the implementation of COI-based metabarcoding for nematodes [21]. Though metabarcoding has been in use for more than a decade [22-24], this method is still under development and standardization for nematode community analysis [21,25-28] due to a lack of reliable quantification methods, PCR biases [29,30], differences in rRNA gene copy numbers among nematode species [31], limited reference databases [21,32], and a lack of consensus on primer sets [21,25,27].

The 18S rRNA gene has been reported to evolve more conservatively than the COI gene and is often used to distinguish between nematode species that are not closely related [33,34]. Though $18 \mathrm{~S}$ rRNA lacks power for the discrimination of closely related nematode species, the presence of conserved regions in the $18 \mathrm{~S}$ rRNA gene has allowed for the design of several promising primer sets for the generic amplification of a wide diversity of nematodes [23,25,35]. The V2, V4, and V9 variable regions of the 18S rRNA gene were suggested to be most suitable for biodiversity assessments after multiple alignment of eukaryotic $18 \mathrm{~S}$ rRNA gene sequences available in the SILVA database [36]. Consequently, the $18 \mathrm{~S}$ rRNA gene may remain the most widely used molecular marker for the identification of nematodes in high throughput sequencing (HTS) approaches [21,37]. An 18S rRNA gene-based HTS approach was proposed as a promising solution for the description of nematode communities from DNA extracted from environmental samples [23]. Likewise, several nematode metabarcoding primer combinations have been designed based on the variable regions of the 18S rRNA gene and used to detect soil nematodes extracted from samples $[21,25,27]$. However, these primer sets were tested on nematodes extracted from soil and not on DNA extracted directly from soil samples. We aimed for a suitable primer set that would amplify and detect soil nematodes from environmental DNA without prior nematode extraction from soil, as well as in the presence of non-target plant, fungal, protist, and metazoan DNA. Therefore, the aim of the present method development study was to compare commonly used primer sets from the literature and a newly designed primer set to identify the most suitable primer set for the metabarcoding of plant-parasitic and free-living soil nematodes. For this, we used DNA extracted from 22 different nematode species, 10 mock communities in water, 20 DNA samples extracted from agricultural fields, 4 root samples with attached soil, and a bulk soil to validate the primer sets.

\section{Materials and Methods}

\subsection{Primer Sets}

We selected four primer sets for the amplicon sequencing of nematode taxa (Table S1 and Figure S1). The primer set SSU_04F/SSU_22R (SSU) amplifies the V1-V2 regions of the 18S rRNA gene and was recently used to describe the assemblages of free-living soil nematodes after nematode isolation from soil using the MiSeq platform [21,26,27]. We designed a primer set, MMS (MMSF: 5'-GGTGCCAGCAGCCGCGGTA-3', MMSR: 5'-CTTTAAGTTTCAGCTTTGC-3') located in the variable V4-V5 regions of the $18 \mathrm{~S}$ rRNA gene. Furthermore, we included the Nemf/18Sr2b primer set (NEM), which was developed for the 454-sequencing platform using a semi-nested PCR approach. In the present study, the second PCR step of the nested PCR was omitted, which resulted in a larger PCR product (around $500 \mathrm{bp}$ ) covering the V6-V8 regions of the $18 \mathrm{~S}$ rRNA gene. We also tested the JB3/JB5 (JB)-targeting of the I3-M11 region of the mitochondrial COI gene in the present study; this primer set was previously used to study nematode communities after initial nematode isolation $[17,21,25]$. 
Several other mitochondrial primer sets, i.e., COX2F/COX2R, COX3F/COX3R [18], JB2/JB5GED [38], and COIF/COIR [39], were initially tested on individual nematode taxa, but due to very low PCR amplification success rates, these primer sets were not included in the subsequent analyses.

\subsection{Nematode Species, Mock Communities and Soil Samples}

In order to test the primer sets, we used DNA extracts from 22 soil nematode species kindly provided by researchers (Table S2). We included plant-parasitic nematodes representing several agriculturally important nematode families (Meloidogynidae, Pratylenchidae, Heteroderidae, and Dolichodoridae) and a free-living nematode taxon belonging to Rhabditidae. We also tested the primer sets on ten mock communities in which the nematode DNA of each specimen was pooled on a volumetric basis (2.5 $\mu \mathrm{L}$ DNA of each taxon) (Table S3).

We used 20 soil samples collected from different fields, as described earlier [40] (Table S4). To represent each soil sample, 20 randomly distributed sites in the field were selected, and $200 \mathrm{~g}$ of upper soil (15 cm soil layer) of each site was sampled and thoroughly mixed by hand before $100 \mathrm{~g}$ subsamples were taken. Samples were freeze-dried for 3 days and subsequently ground in a mixer mill (Retsch MM301, Haan, Germany) for $10 \mathrm{~min}$. We used $0.25 \mathrm{~g}$ of soil for DNA extraction from the thoroughly ground and homogenized $100 \mathrm{~g}$ soil samples.

\subsection{Root Samples}

We grew 4 different Arabidopsis thaliana lines (N6567, N8034, N929, and NW25) to test primer efficiency in detecting soil nematodes in roots (including attached soil). We filled 20 pots each with $400 \mathrm{~g}$ of homogenized clayey sandy soil $(\mathrm{pH}=5.9)$, and we transplanted three-week old 5 Arabidopsis seedlings into each pot. Four pots were filled with soil and kept without plants-these were considered bulk soil (BS). Pots were placed randomly in a glasshouse at $22-24^{\circ} \mathrm{C}$ and $12 \mathrm{~h} / 12 \mathrm{~h}$ light-dark conditions. After 5 weeks, roots were harvested by gently pressing the sides of pots to loosen soils around the plant before careful uprooting. We also took a bulk soil sample $(10 \mathrm{~g})$ with 4 biological replicates. Roots were shaken gently to remove loosely adhering soil. The five plant roots from each pot were pooled to represent a biological replicate. Four biological replicates of each Arabidopsis line were harvested. The roots were quickly transferred to collecting tubes and plunged into liquid nitrogen. All samples were transferred to a $-80^{\circ} \mathrm{C}$ freezer until further use. Before DNA extraction, bulk soil and root samples were freeze-dried before grinding in a Geno/Grinder 2000 (RAMCON, Denmark) at $1500 \mathrm{rpm}$ for $6 \times 1 \mathrm{~min}$.

\subsection{DNA Extraction, PCR and Sequencing Library Preparation}

DNA was extracted from $0.25 \mathrm{~g}$ of each of the ground soil and root samples using the PowerLyzer soil DNA extraction kit (Qiagen, Hilden, Germany) according to the manufacturer's instructions, except that samples were homogenized in a Geno/Grinder 2000 (RAMCON, Denmark) at 1500 rpm for $3 \times 30 \mathrm{~s}$. DNA concentrations were measured using a Qubit Fluorometer (Thermo Fisher Scientific, Waltham, MA, USA) and diluted to $2 \mathrm{ng} / \mu \mathrm{L}$ in each soil and root sample.

To amplify target regions, PCR was performed in a $25 \mu \mathrm{L}$ reaction mixture consisting of $5 \mu \mathrm{L}$ of a Promega 5X reaction buffer (Promega Corporation, Madison, WI, USA), $1.5 \mu \mathrm{L}$ of $\mathrm{MgCl}_{2}(25 \mathrm{mM}), 2 \mu \mathrm{L}$ of dNTPs $(2.5 \mathrm{mM}), 0.5 \mu \mathrm{L}$ of each primer $(10 \mu \mathrm{M}), 0.125 \mu \mathrm{L}$ of GoTaq Flexi polymerase (5U, Promega Corporation, Madison, WI, USA), and $2 \mu \mathrm{L}$ of a DNA template $(1 \mathrm{ng} / \mu \mathrm{L}$ for individual nematode species and mock communities; $2 \mathrm{ng} / \mu \mathrm{L}$ for soil and root samples). All four primer sets were tested on individual taxa, mock, and soil samples. In addition, the MMS and NEM primer sets were used on root samples. PCR cycles for the JB primer combination were $94{ }^{\circ} \mathrm{C}$ for $5 \mathrm{~min}\left(94{ }^{\circ} \mathrm{C}\right.$ for $1 \mathrm{~min}, 50{ }^{\circ} \mathrm{C}$ for $30 \mathrm{~s}$, and $72{ }^{\circ} \mathrm{C}$ for $\left.45 \mathrm{~s}\right) 35$ cycles, $72{ }^{\circ} \mathrm{C}$ for $10 \mathrm{~min}$, and $4{ }^{\circ} \mathrm{C}$ on hold [16]. Similar PCR cycles were used for the other sets, except that the annealing temperature was set to $53^{\circ} \mathrm{C}$ for MMS and NEM and set to $55^{\circ} \mathrm{C}$ for the SSU primer set [41]. Each of the primer sets of the first PCR (Table S1) were tagged 
with the Illumina adapter overhang nucleotide sequence. PCR products of the first PCR were diluted (1:5) and pooled for each sample.

A second PCR was performed for dual indexing to enable the pooling of all samples. The master mix of this PCR was identical to the first PCR except that $2 \mu \mathrm{L}$ of the DNA template and $2 \mu \mathrm{L}$ of the index primer combinations were used. Each index primer consisted of a specific sequence for Illumina sequencing, a unique 8 bp multiplex identifier, and the Illumina adapter overhang sequence. The second PCR was performed with the following cycles: $94{ }^{\circ} \mathrm{C}$ for $5 \mathrm{~min},\left(94{ }^{\circ} \mathrm{C}\right.$ for $30 \mathrm{~s}, 55^{\circ} \mathrm{C}$ for $30 \mathrm{~s}$, and $72{ }^{\circ} \mathrm{C}$ for $\left.1 \mathrm{~min}\right) 13$ cycles, $72{ }^{\circ} \mathrm{C}$ for $10 \mathrm{~min}$, and $4{ }^{\circ} \mathrm{C}$ on hold. All amplicons were visualized by gel electrophoresis, pooled, precipitated, and the pellet dissolved in DNAse free water. Pooled DNA was run on a gel, and amplicons were excised and purified using the QIAquick Gel Extraction kit (Qiagen, Hilden, Germany) according the manufacturer's instructions. Finally, the DNA concentrations were measured by Qubit Fluorometer (Thermo Fisher Scientific, Waltham, MA, USA), and DNA libraries were sent for sequencing on an Illumina MiSeq sequencer with PE300 (Eurofins Genomics, Ebersberg, Germany).

\subsection{Sequence Analysis}

Sequences obtained from the Illumina MiSeq run were demultiplexed using a demultiplexer [42]. In brief, paired-end reads were joined using VSEARCH version 2.6 of QIIME2 [43]. To join the paired-end reads, we used a default overlapping minimum read length of 10 base pairs and removed reads with quality Phred scores of $<30$. Internal barcodes, forward and reverse primers, and reads less than 250 base pairs were also excluded. Following this, sequences were dereplicated and screened for chimera detection and clustered at a 99\% similarity level using VSEARCH version 2.6. Taxonomy assignments for the clustered operational taxonomic units (OTUs) were done in the SILVA 132 reference database for $18 S[44,45]$ and the MIDORI curated database for mitochondrial-encoded sequence reads using assign_taxonomy.py [46]. Moreover, all nematode OTUs were queried against the NCBI GenBank database to reconfirm their taxonomic assignments.

\subsection{Statistical Analysis}

Community composition was determined using OTUs from a bulk soil and root samples. We used phyloseq R packages for diversity-based calculations [47]. The OTU table was transformed before the calculation of relative abundance and rarified before OTU richness and alpha diversity measure determination. Beta diversity-based dissimilarities matrices on nematode communities for the partitioning of variance was calculated using PERMANOVA and Adonis test in R statistics.

\section{Results}

\subsection{Data Characteristics}

We analyzed sequence reads from the 22 nematodes taxa, 10 mock communities and 20 soils using the four primer sets. After quality control, sequence reads were clustered into 95, 534, 273, and 258 Nematoda OTUs for JB (JB3/JB5), SSU (SSU_04F/SSU_22R), MMS (MMSF/MMSR), and NEM $(\mathrm{Nemf} / 18 \mathrm{Sr} 2 \mathrm{~b})$ primer sets, respectively. The sequence reads of root samples were clustered into 58 and 126 Nematoda OTUs for the MMS and NEM primer sets, respectively.

\subsection{Individual Nematode Species}

For the individual nematode species, we could assign 8 of the 22 species to species rank and 10 to genus rank using the JB primer set, whereas four species were not amplified (Table 1). Using the SSU primer set, only 12 out of the 22 species were amplified, of which seven were assigned to genus rank and five to species rank. The MMS primer set amplified all nematodes species except for Meloidogyne graminicola (Table 1). This primer set identified Meloidogyne at the genus rank except for Meloidogyne hapla, which was assigned at the species rank. Five nematode taxa were also assigned at the 
species rank. The NEM primer set successfully amplified all the root knot nematode species except for M. graminicola. Most Meloidogyne species were assigned to the genus rank except for Meloidogyne hapla, which was assigned to the species level. Four nematode taxa were assigned at the species rank by the NEM primer set. Cyst nematodes (Heterodera carotae and Heterodera schachtii) were assigned to the family rank with the NEM primer set (Table 1). The root lesion nematodes Pratylenchus spp. were detected as Pratylenchus neglectus and Pratylenchus penetrans by both the JB and NEM primer sets; MMS detected these nematodes as Pratylenchus neglectus and Pratylenchus sp., while the SSU primer set did not amplify root lesion nematodes.

Table 1. The efficiency of four metabarcoding primer sets in the detection of individual nematode taxa at different taxonomic ranks using Illumina sequencing.

\begin{tabular}{|c|c|c|c|c|}
\hline Nematode Taxa & JB & SSU & MMS & NEM \\
\hline Meloidogyne incognita & Genus & Not detected & Genus & Genus \\
\hline Meloidogyne arenaria & Genus & Genus & Genus & Genus \\
\hline Meloidogyne javanica & Genus & Genus & Genus & Genus \\
\hline Meloidogyne graminicola & Genus & Not detected & Not detected & Not detected \\
\hline Meloidogyne ethiopica & Genus & Genus & Genus & Genus \\
\hline Meloidogyne inornata & Genus & Genus & Genus & Genus \\
\hline Meloidogyne ulmi & Genus & Genus & Genus & Genus \\
\hline Meloidogyne luci & Genus & Genus & Genus & Genus \\
\hline Meloidogyne hapla & Species & Genus & Species & Species \\
\hline Meloidogyne enterolobii & Species & Not detected & Genus & Genus \\
\hline Meloidogyne chitwoodi & Species & Not detected & Genus & Genus \\
\hline Meloidogyne fallax & Genus & Not detected & Genus & Genus \\
\hline Meloidogyne minor & Genus & Species & Genus & Genus \\
\hline Meloidogyne naasi & Species & Not detected & Genus & Genus \\
\hline Pratylenchus penetrans & Species & Not detected & Genus & Species \\
\hline Pratylenchus spp. & Species & Not detected & Species & Species \\
\hline Heterodera carotae & Not detected & Not detected & Genus & Family \\
\hline Heterodera schachtii & Species & Species & Genus & Family \\
\hline Belonolaimus longicaudatus & Not detected & Species & Species & Species \\
\hline Bursaphelenchus mucronatus & Not detected & Not detected & Genus & Genus \\
\hline Caenorhabditis elegans & Species & Species & Species & Species \\
\hline Ditylenchus dipsaci & Not detected & Species & Species & Genus \\
\hline
\end{tabular}

The NCBI Blast tool was used for taxonomic assignments, and top hits with $100 \%$ coverage, and $100 \%$ sequence similarities at the species rank and $\geq 99 \%$ at the genus rank, were considered. JB: JB3/JB5; SSU: SSU_04F/SSU_22R; MMS: MMSF/MMSR; NEM: Nemf/18Sr2b.

\subsection{Mock Communities}

In mock communities, JB-generated OTUs were assigned to the genus rank within the Meloidogynidae family, except for Meloidogyne hapla and Meloidogyne naasi, which were assigned to the species rank, and Meloidogyne minor, which was not amplified (Table 2). The cyst nematode taxa Globodera pallida, Globodera rostochiensis, and Heterodera schachtii were detected at the species rank, while Heterodera carotae was not amplified. Taxa within Pratylenchidae and Rhabditidae were assigned at the species rank, and Belonolaimus longicaudatus was not detected (Table 2).

The SSU-generated OTUs were assigned to the genus rank within the Meloidogynidae, except for three taxa (Meloidogyne minor, Meloidogyne hapla, and Meloidogyne inornata) that were not amplified. Three taxa within Heteroderidae were detected at the genus rank, while Globodera pallida was assigned to the species rank. Caenorhabditis elegans was also assigned to the species rank. The SSU primers failed to amplify Pratylenchus penetrans and Belonolaimus longicaudatus in any mock community sample (Table 2).

The MMS-generated OTUs from ten taxa of the Meloidogynidae were assigned to the genus rank, while Meloidogyne hapla was assigned to the species rank and Pratylenchus penetrans was not detected in mock communities. The OTUs belonging to the taxa Globodera pallida, Heterodera schachtii, and Caenorhabditis elegans were assigned to the species rank (Table 2). 
The NEM primer set amplified and detected nematode taxa of Meloidogynidae in accordance with the MMS primer pair; Pratylenchus penetrans, Belonolaimus longicaudatus, and Caenorhabditis elegans were assigned at the species level; the Globodera species were assigned at the genus rank; and the two Heterodera species were assigned at the family rank (Table 2).

Table 2. The efficiency of four metabarcoding primers in the detection of taxa in mock communities using Illumina sequencing.

\begin{tabular}{lcccc}
\hline Taxa in Mock Communities & JB & SSU & MMS & NEM \\
\hline Meloidogyne incognita & Genus & Genus & Genus & Genus \\
Meloidogyne arenaria & Genus & Genus & Genus & Genus \\
Meloidogyne ethiopica & Genus & Genus & Genus & Genus \\
Meloidogyne inornata & Genus & Not detected & Genus & Genus \\
Meloidogyne ulmi & Genus & Genus & Genus & Genus \\
Meloidogyne luci & Genus & Genus & Genus & Genus \\
Meloidogyne hapla & Species & Not detected & Species & Species \\
Meloidogyne chitwoodi & Genus & Genus & Genus & Genus \\
Meloidogyne fallax & Genus & Genus & Genus & Genus \\
Meloidogyne minor & Not detected & Not detected & Genus & Genus \\
Meloidogyne naasi & Species & Genus & Genus & Genus \\
Pratylenchus penetrans & Species & Not detected & Not detected & Species \\
Heterodera carotae & Not detected & Genus & Genus & Family \\
Heterodera schachtii & Species & Genus & Species & Family \\
Belonolaimus longicaudatus & Not detected & Not detected & Species & Species \\
Caenorhabditis elegans & Species & Species & Species & Species \\
Globodera pallida & Species & Species & Species & Genus \\
Globodera rostochiensis & Species & Genus & Genus & Genus \\
\hline
\end{tabular}

The NCBI Blast tool was used for taxonomic assignments, and top hits with $100 \%$ coverage, as well as 100\% sequence similarities at the species rank and $\geq 99 \%$ at the genus rank, were considered. JB: JB3/JB5; SSU: SSU_04F/SSU_22R; MMS: MMSF/MMSR; NEM: Nemf/18Sr2b.

\subsection{Detection of Nematodes in Soil Samples}

For the JB primer set, only $4 \%$ of the total sequence reads from the soil samples were classified as Nematoda (Figure 1). For the SSU primer set, only $1 \%$ of the sequence reads from the soil samples were classified as Nematoda (Figure 1). This primer set amplified a broad spectrum of other eukaryotes such as fungi, plant, Cercozoa, and Charophyta. For the newly designed primer set (MMS), $14 \%$ of total sequence reads belonged to Nematoda, and for the NEM primer set, $74 \%$ of the total sequence reads were assigned to Nematoda in the soil samples (Figure 1).

There was a low recovery of sequence reads that assigned to Nematoda from soil samples using the JB and SSU primer sets (Figure 1, Figure S2, and Table S5). In contrast, the NEM and MMS primers detected a wide range of nematode taxa from different families from the $0.25 \mathrm{~g}$ soil samples (Table S5). Twenty nematode families were detected using both the NEM and MMS primer sets, and seven families were uniquely detected by each primer set (Figure 2A). We recorded 13 and 12 different unique genera in soil samples by the NEM and MMS primer sets, respectively, and 25 genera were detected by both primer sets (Figure 2A). We detected 29 and 20 unique nematode species with the NEM and MMS primer sets, respectively, while 23 species were detected by both primer sets (Figure 2A). These two primers sets were able to detect a high diversity of nematode taxa in the soil samples compared to the JB and SSU primer sets (Figures S2 and S3). 


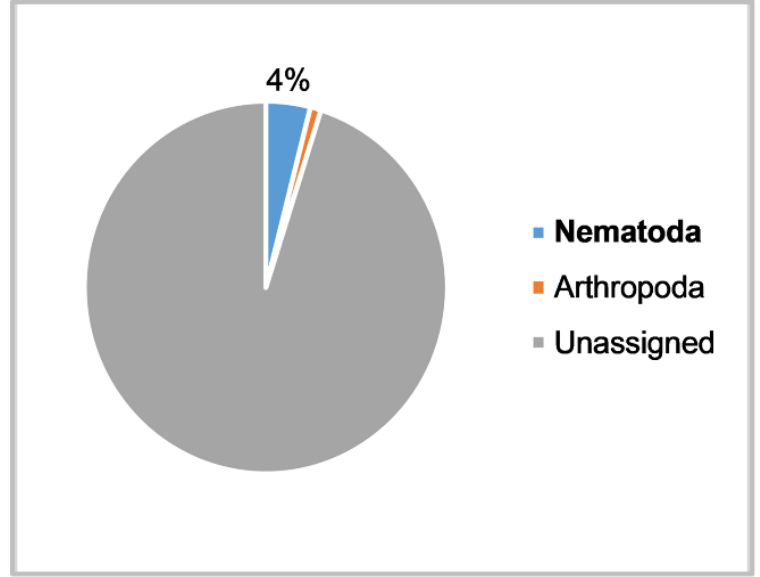

(A)

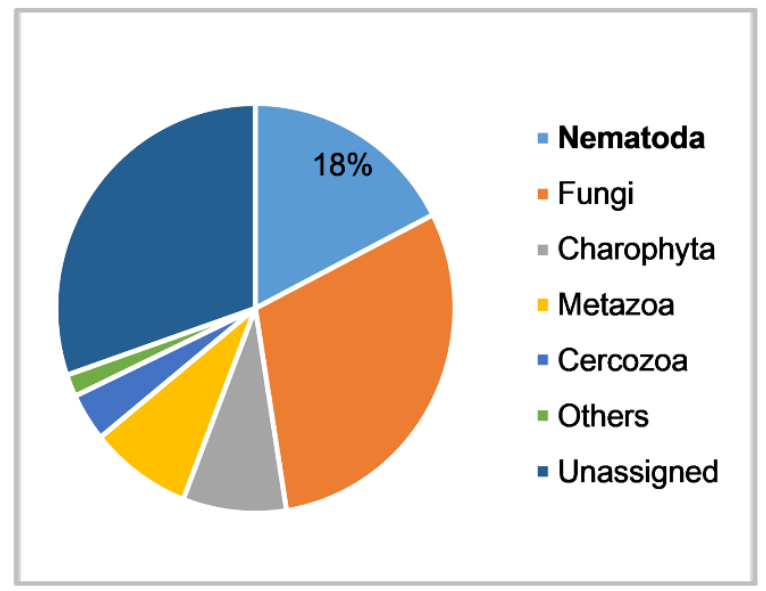

(C)

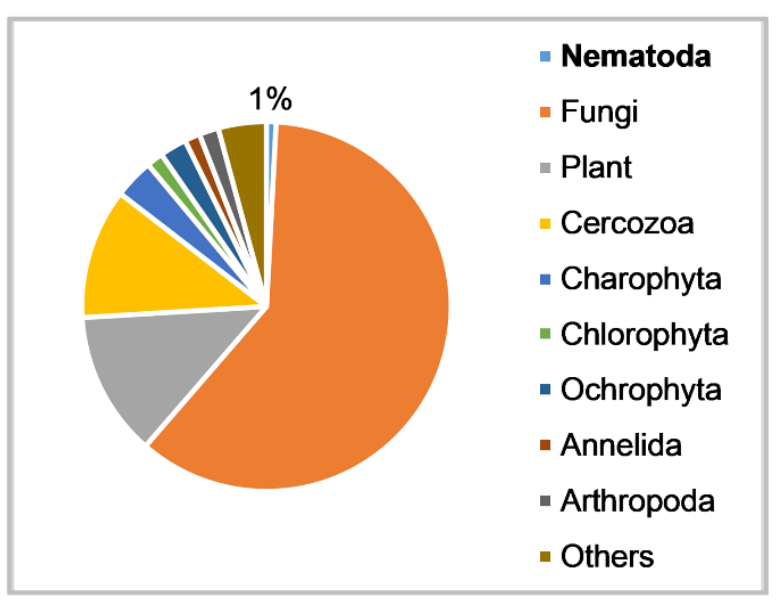

(B)

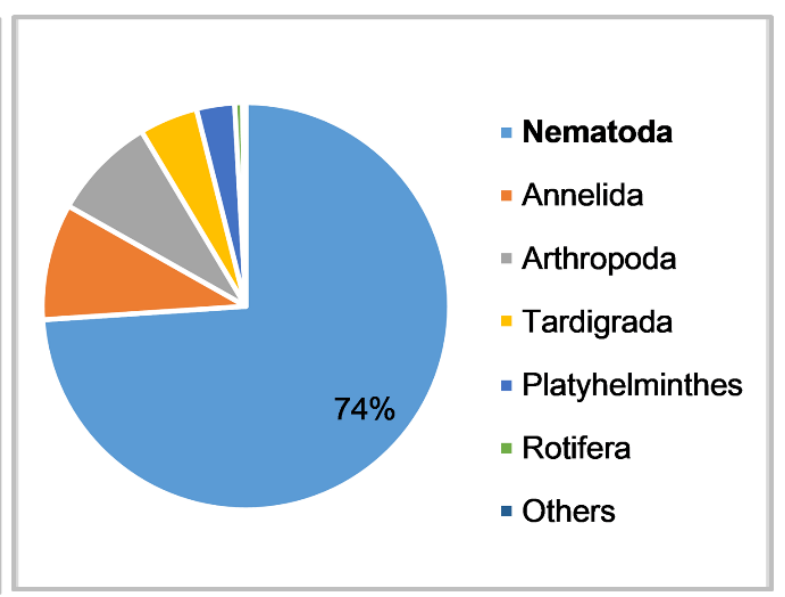

(D)

Figure 1. Relative distribution of sequence reads in soil samples amplified with JB: JB3/JB5 (A), SSU: SSU_04F/SSU_22R (B), MMS: MMSF/MMSR (C), and NEM: Nemf/18Sr2b (D) primer sets; percentage indicates the proportion of sequence reads that were assigned to Nematoda.

\subsection{Nematode Communities in Root Samples}

Next, we tested the efficiencies of the NEM and MMS primers in the amplification and detection of nematodes associated with Arabidopsis roots and a bulk soil sample. Due to the low performance of the JB and SSU primer sets in the above analyses, we did not include these primers in the subsequent analysis of primer performance in the soil and root samples. In the root samples, we recovered 30 families, 35 genera, and 51 species with the NEM primers, whereas the MMS primers detected 15 families, 21 genera, and 31 species (Figure 2B). All 15 families detected by the MMS primers were also detected by the NEM primers, whereas 15 unique families were only detected with the NEM primers (Figure 2B). Sixteen genera were uniquely detected by NEM, whereas only two genera were unique to MMS and 19 genera were detected by both primers sets. We detected 28 unique nematode species with the NEM primer set, while only eight unique species were detected by the MMS primer set and 23 species were detected by both primer sets (Figure 2B). 


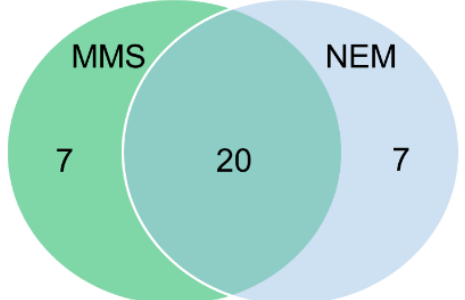

Families

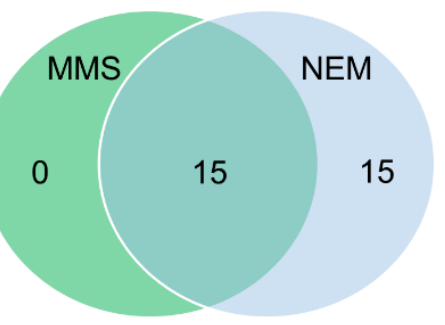

Families

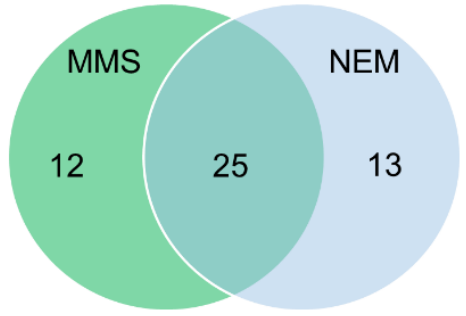

Genera

(A)

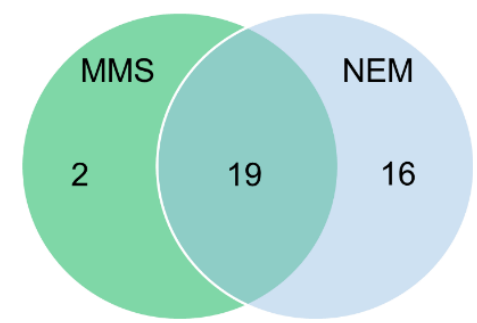

Genera

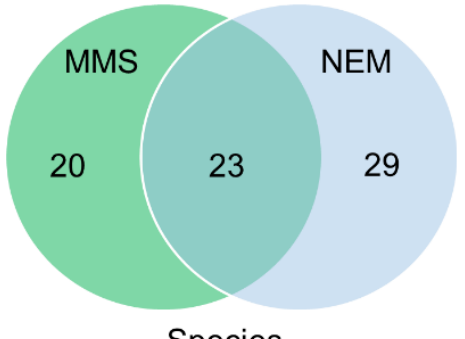

Species

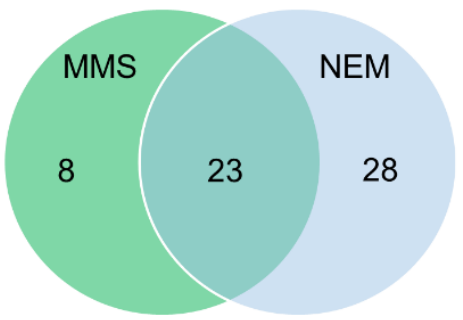

Species

(B)

Figure 2. Venn diagrams showing the number of nematode taxa detected in soil (A) and root samples (B) by the MMS (MMSF/MMSR) and NEM (Nemf/18Sr2b) primer sets.

Both primer sets detected plant-parasitic and free-living nematode taxa in the bulk soil and root samples (Figure 3). The NEM primer set detected a higher number of nematode taxa compared to the MMS primer set (Table S6). The relative abundance of two important plant-parasitic taxa Meloidogyne and Pratylenchus in root samples were comparatively lower when using the MMS primer set compared to the NEM primer set (Figure 3A,B). Two taxa, Mesorhabditis and Oscheius, belonging to Rhabditidae appeared among the ten most abundant taxa in MMS dataset, whereas these taxa were not found among the ten most abundant genera in the NEM dataset (Figure 3A,B). However, the NEM primer set was able to detect Mesorhabditis belari, and Oscheius tipulae in root samples (Table S6). The dataset generated by the NEM primer set had a higher observed OTU richness and Shannon diversity indices compared to the MMS primer set (Figure 3C,D). The NEM primer set explained $68 \%$ of the variation of nematode communities ( $p<0.001)$, whereas $59 \%$ of the variation $(p<0.001)$ was explained by the MMS primer set in Principal Coordinates Analysis (PCoA) plots (Figure 3E,F and Tables S7 and S8). 

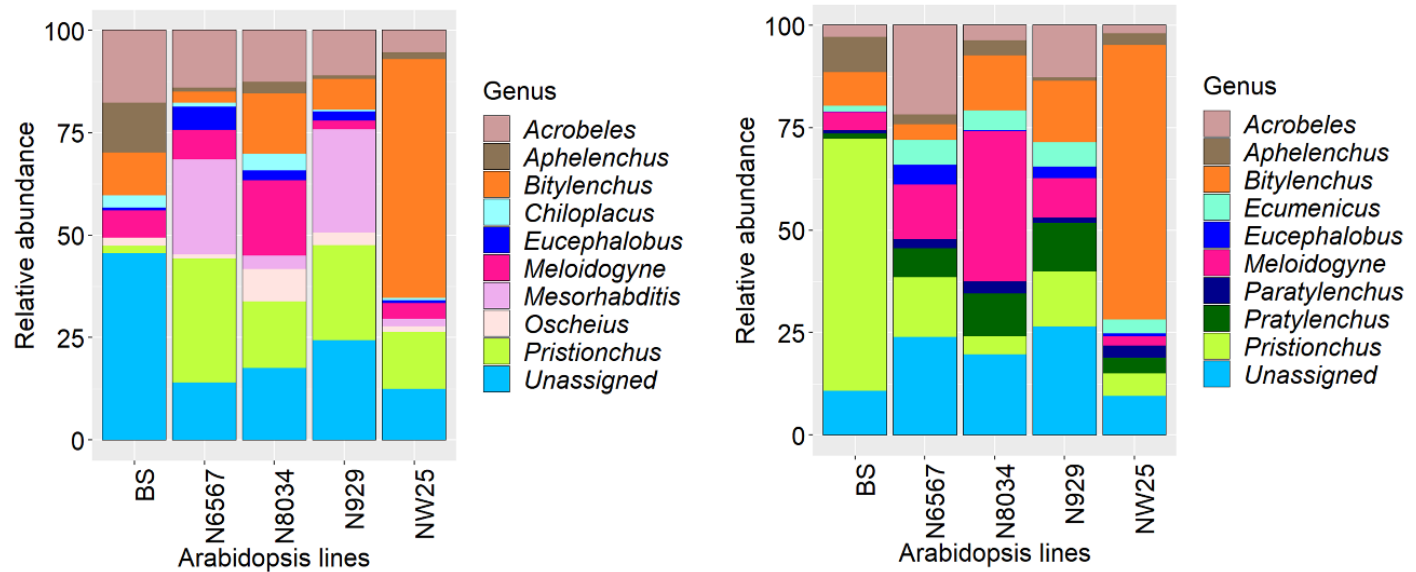

(A)

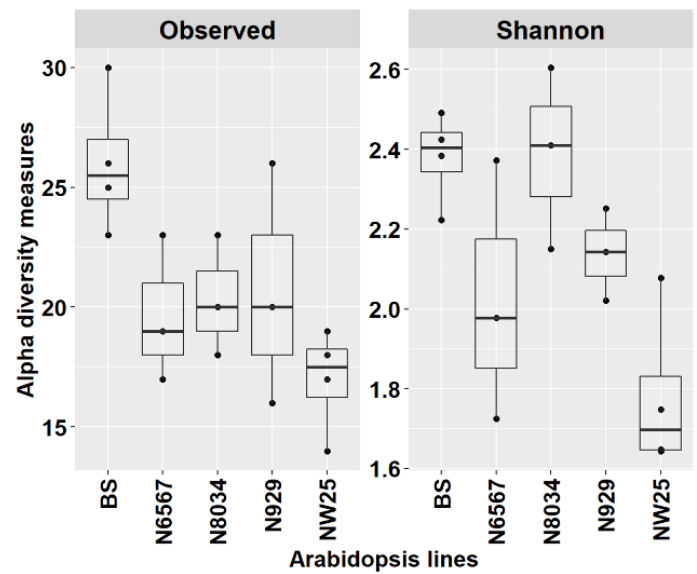

(C)

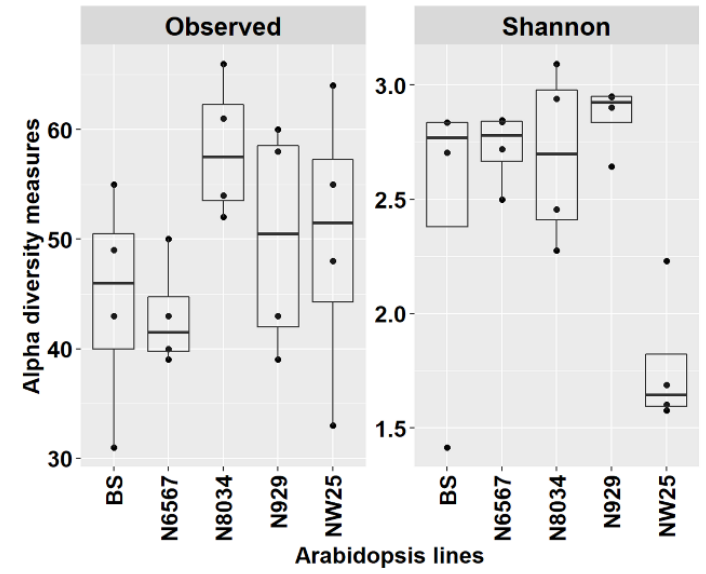

(D)

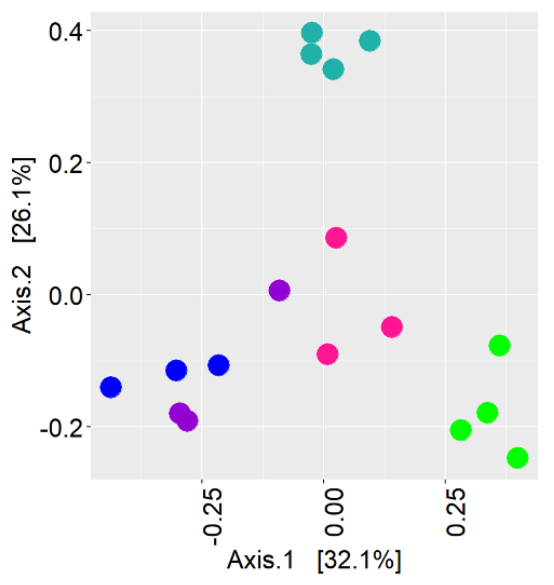

(E)
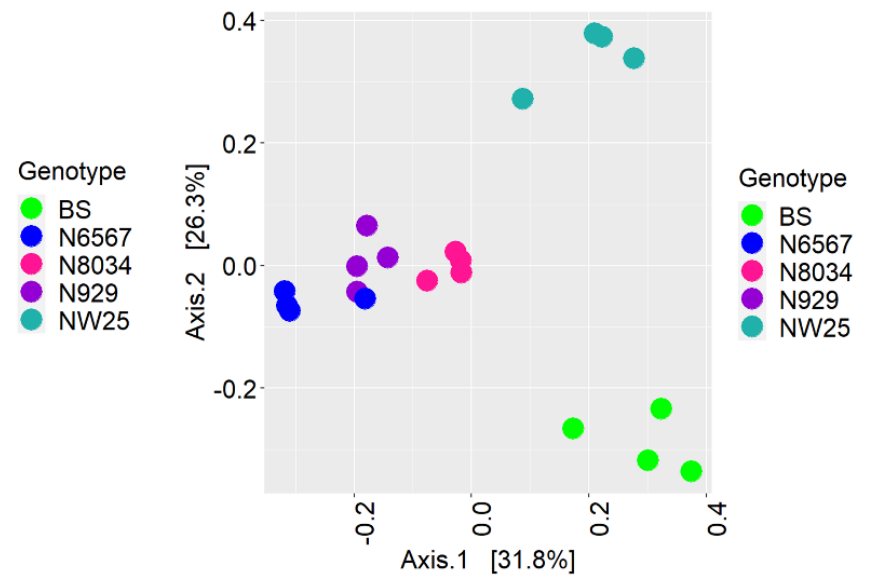

(F)

Figure 3. Comparison of nematode taxonomic profiles from the roots of different Arabidopsis accessions (N6567, N8034, N929, and NW25) and unplanted bulk soil (BS) amplified using MMS: MMSF/MMSR $(\mathbf{A}, \mathbf{C}, \mathbf{E})$ and NEM: Nemf/18Sr2b (B,D,F) primer sets. (A,B) Relative abundance of the ten most abundant genera are shown. (C,D) The alpha diversity was estimated using observed operational taxonomic unit (out) richness and Shannon diversity indices. (E,F) Principal Coordinates Analysis (PCoA) ordination plot using Bray-Curtis distance matrices.

\section{Discussion}

Generally, HTS-based studies of nematode diversity include a nematode extraction step to increase nematode target DNA in the background of DNA from other soil-living organisms due to a lack of 
nematode-specific primer pairs [21,25-27,48]. Such nematode extraction steps may not be practical in studies of plant-nematode interactions where only small amounts of samples are usually available. We previously developed an amplification strategy for 454 pyrosequencing that selectively amplifies nematode DNA from total soil DNA extractions [35]. In the present study, we modified and adapted this amplification strategy based on the NEM primer set for sequencing on the Illumina MiSeq platform. We compared the NEM primer set with two widely used primer sets and a novel primer set developed in this study for their ability to selectively amplify and detect nematode taxa. In our study, the mock communities were mainly composed of plant-parasitic nematode taxa to test the applicability of the primer sets in detecting and distinguishing between plant-parasitic nematode taxa. To further explore the suitability and efficiency of the primer sets to study nematode communities including non-parasitic nematode taxa, we tested the primers on mixed communities in a range of soil samples.

The COI gene-based primer set was initially considered as a suitable marker for barcoding purposes due to its ability to discriminate closely related species in animal phyla [12], as well as its high interspecific and low intraspecific genetic variation in the Nematoda phylum [16]. We found that the COI-targeting JB primer set worked to some extent on extracted nematodes. Hence, JB primers amplified individual nematode species and nematode taxa in mock communities. However, the performance of this primer set using DNA from soil samples was poor; hence, around $95 \%$ of the OTUs were not assigned to any taxonomic level. BLAST searches confirmed that these unassigned OTUs did not belong to the Nematoda phylum. Moreover, only four nematode genera and seven species were detected by the JB primer set in soil. As previously suggested by other researchers, the COI gene has high mutation rates and primer sequences are poorly conserved throughout the Nematoda phylum [14,49]. Based on the present study of individual nematode taxa, mock communities, and soil samples without prior nematode extraction, we found that the JB primer set targeting the I3-M11 partition of the COI gene is not suitable for nematode metabarcoding, especially in a soil DNA background.

In a recent study, the small subunit ribosomal RNA gene based SSU primer set (SSU_04F/SSU_22R) outperformed the mitochondrial COI gene based primer set (JB3/JB5GED) for nematode species and genus level detection on extracted nematode samples [21,25]. However, in our study, several agriculturally important nematode taxa were not amplified with the SSU primer set. Furthermore, this primer set resulted in only $1 \%$ Nematoda reads from the soil DNA samples. This is supported by earlier studies, where this SSU primer set amplified a large number of non-nematode reads from environmental marine sediment samples [22,50-52]. In the soil samples, the SSU primer set only detected 23 nematode genera compared to the 37 and 38 different genera detected with MMS and NEM primer sets, respectively. Therefore, this primer set is not suitable for targeting nematode diversity without an initial nematode extraction step. The analysis of the individual nematode taxa showed that a better taxonomic resolution was achieved with the MMS primer set that targets the V4-V5 regions of the $18 \mathrm{~S}$ rRNA gene compared to JB and SSU primer sets. The efficiency of this primer set was further tested using mock communities, in which it was possible to detect all nematode taxa except for Pratylenchus penetrans. The MMS primer set detected a high diversity of the nematode communities in the soil samples. However, the primer set detected fewer nematode taxa in the plant root samples compared to the NEM primer set. Additionally, we detected a large number of reads from plants in root samples with the MMS primer set, which further confirmed its poor performance on root samples. This was probably due to competition in primer annealing between nematode and plant DNA templates due to the conserved region in the 18S rRNA gene, even between nematodes and plants.

Most of the nematode taxa in the test of individual nematode species and in the mock communities were amplified and detected using the NEM primer set. This primer set also detected a wide range of nematode taxa in the different soil DNA samples. Furthermore, the NEM primer set amplified 51 nematode species, compared to 31 species by the MMS primer set, in the root samples. Importantly, the NEM primer set efficiently amplified nematode DNA in the presence of plant DNA, thus indicating that this primer set has a higher selectivity in favor of nematode DNA compared to the MMS primer set and is thus promising for root-nematode interaction studies. 
The sequence reads from the Rhabditidae family (notably Mesorhabditis) were much more relatively abundant in the MMS than in the NEM-generated dataset in the root samples. This discrepancy could have been due to a three-nucleotide mismatch between the 18Sr2b primer of the NEM primer set and the Mesorhabditis DNA template. A multiple sequence alignment of 60 different accessions of Rhabditidae (Figure S4) showed that most of the taxa of this family did not show any mismatch except for the three genera (Mesorhabditis, Pelodera, and Rhabditis).

In the present study, different field soils were used to compare the suitability of the metabarcoding primer sets for the detection of nematode diversity in total soil DNA extractions without prior nematode extraction. For DNA extraction, we only used $0.25 \mathrm{~g}$ of soil out of the thoroughly homogenized $100 \mathrm{~g}$ subsamples that had been collected under the assumption that the nematode DNA was evenly distributed after the homogenization. If the preservation of diversity should be ensured, we suggest to do several DNA extractions from each soil sample. However, we were still able to detect a large nematode diversity using the NEM primer pair when using this small sample size. The retrieval of a high nematode diversity in only $0.25 \mathrm{~g}$ homogenized soil samples shows that the employed metabarcoding strategy is applicable in, e.g., rhizosphere community studies where the total sample weight rarely exceeds a few grams.

\section{Conclusions}

Both the MMS and NEM primer sets outperformed the JB and SSU primer sets based on the nematode detection of individual nematode taxa, mock communities, and soil samples. A comparison of the MMS and NEM primer sets in root samples suggested that the NEM primer set provided a better representation of the nematode community structure in the studied samples. Consequently, we propose the use of the NEM primer set for the detection of nematode taxa on DNA directly extracted from soil, root, or rhizosphere soil samples. An assignment of lower Linnaean taxonomies (genus and species) to sequence reads is a crucial step in the use of DNA markers for biodiversity assessment. Therefore, we conclude that the NEM primer set efficiently detects diverse nematode families and can efficiently detect nematodes at the genus and, in some cases, species ranks in root/rhizosphere soil samples.

Supplementary Materials: The following are available online at http://www.mdpi.com/1424-2818/12/10/388/s1, Figure S1: Location of metabarcoding primers targeting variable regions in mitochondrial (A) and 18S rRNA (B) gene used in the present study. Figure S2. Relative abundance of the nematode genera in soil samples amplified by the JB primer set (A) and SSU primer set (B). Here, unassigned sequence reads were junk DNA, which confirmed by BLAST search. Figure S3. Relative abundance of the nematode genera in soil samples amplified by MMS primer set (A) and NEM primer set (B). Figure S4. Multiple sequence alignment of MMS (A: MMSF) and NEM (B: 18Sr2b) primer sets and representative taxa of Rhabditidae. Table S1: Metabarcoding primer sets used in the present study. Table S2. List of individual nematode species, donating institutes and nematode families of the species used in the study. Table S3. Composition of mock communities used in the study. Table S4. Cropping history and soil properties of twenty different soils used in the study. Table S5. Efficiency of four metabarcoding primers in detection of soil nematodes in twenty different soils at lower taxonomic rank. Table S6. Efficiency of two metabarcoding primers in detection of soil nematodes in roots including background soil. Table S7. Adonis test Permutation analysis of variance (PERMANOVA) for Arabidopsis lines and background soil by MMS primer set. Adonis test was based on Bray-Curtis distance matrices for nematode community dissimilarity assessment using 10,000 permutations. Table S8. Adonis test Permutation analysis of variance (PERMANOVA) for Arabidopsis lines and background soil by NEM primer set. Adonis test was based on Bray-Curtis distance matrices for nematode community dissimilarity assessment using 10,000 permutations.

Data Availability: Bioinformatics pipeline, R code, and associated protocols are available from the corresponding author on reasonable request.

Author Contributions: Conceptualization, M.M.S., R.S., M.N., M.V., and T.K.; methodology, M.M.S., R.S., and M.N.; software, M.M.S. and R.S.; formal analysis, R.S. and M.M.S.; investigation, M.M.S.; data curation, M.M.S. and R.S.; writing_-original draft preparation, M.M.S.; writing—review and editing, M.M.S., M.N., T.K., M.V., and R.S.; visualization, M.M.S.; supervision, M.N., T.K., and M.V.; project administration, M.N., T.K., and M.V.; funding acquisition, M.M.S. and M.N. All authors have read and agreed to the published version of the manuscript.

Funding: This research was funded by Aarhus University, Denmark, Project Number: 27747. 
Acknowledgments: We are grateful to Andrea M. Skantar, United State Department of Agriculture, and Barbara Geric Stare, Sasa Sirca, and Gregor Urek, Department of Plant Protection, Agricultural Institute of Slovenia, for contributing DNA samples of nematode species. We would like to thank Susana Santos for data visualization. We would also like to thank Mathilde Schiøtt Dige and Simone Ena Rasmussen for their excellent laboratory assistance.

Conflicts of Interest: The authors declare no conflict of interest.

\section{References}

1. Lambshead, P.J.D.; Boucher, G. Marine nematode deep-sea biodiversity-hyperdiverse or hype? J. Biogeogr. 2003, 30, 475-485. [CrossRef]

2. Van den Hoogen, J.; Geisen, S.; Routh, D.; Ferris, H.; Traunspurger, W.; Wardle, D.A.; De Goede, R.G.M.; Adams, B.J.; Ahmad, W.; Andriuzzi, W.S.; et al. Soil nematode abundance and functional group composition at a global scale. Nat. Cell Biol. 2019, 572, 194-198. [CrossRef] [PubMed]

3. Agatha, S.; Strüder-Kypke, M.C. Phylogeny of the order Choreotrichida (Ciliophora, Spirotricha, Oligotrichea) as inferred from morphology, ultrastructure, ontogenesis, and SSrRNA gene sequences. Eur. J. Protistol. 2007, 43, 37-63. [CrossRef] [PubMed]

4. De Ley, P.; De Ley, I.T.; Morris, K.; Abebe, E.; Mundo-Ocampo, M.; Yoder, M.; Heras, J.; Waumann, D.; Rocha-Olivares, A.; Burr, A.J.; et al. An integrated approach to fast and informative morphological vouchering of nematodes for applications in molecular barcoding. Philos. Trans. R. Soc. B Biol. Sci. 2005, 360, 1945-1958. [CrossRef] [PubMed]

5. Geiger, M.; Astrin, J.; Borsch, T.; Burkhardt, U.; Grobe, P.; Hand, R.; Hausmann, A.; Hohberg, K.; Krogmann, L.; Lutz, M.; et al. How to tackle the molecular species inventory for an industrialized nation-Lessons from the first phase of the German Barcode of Life initiative GBOL (2012-2015). Genome 2016, 59, 661-670. [CrossRef] [PubMed]

6. Abebe, E.; Mekete, T.; Thomas, W. A critique of current methods in nematode taxonomy. Afr. J. Biotechnol. 2013, 10, 312-323. [CrossRef]

7. Blaxter, M.; Mann, J.; Chapman, T.; Thomas, F.; Whitton, C.; Floyd, R.; Abebe, E. Defining operational taxonomic units using DNA barcode data. Philos. Trans. R. Soc. B Biol. Sci. 2005, 360, 1935-1943. [CrossRef] [PubMed]

8. Holterman, M.; Holovachov, O.; Elsen, S.V.D.; Van Megen, H.; Bongers, T.; Bakker, J.; Helder, J. Small subunit ribosomal DNA-based phylogeny of basal Chromadoria (Nematoda) suggests that transitions from marine to terrestrial habitats (and vice versa) require relatively simple adaptations. Mol. Phylogenet. Evol. 2008, 48, 758-763. [CrossRef]

9. Holterman, M.; Karssen, G.; Elsen, S.V.D.; Van Megen, H.; Bakker, J.; Helder, J. Small Subunit rDNA-Based Phylogeny of the Tylenchida Sheds Light on Relationships Among Some High-Impact Plant-Parasitic Nematodes and the Evolution of Plant Feeding. Phytopathology 2009, 99, 227-235. [CrossRef]

10. Kiewnick, S.; Holterman, M.; Elsen, S.V.D.; Van Megen, H.; Frey, J.E.; Helder, J. Comparison of two short DNA barcoding loci (COI and COII) and two longer ribosomal DNA genes (SSU \& LSU rRNA) for specimen identification among quarantine root-knot nematodes (Meloidogyne spp.) and their close relatives. Eur. J. Plant Pathol. 2014, 140, 97-110. [CrossRef]

11. Floyd, R.; Abebe, E.; Papert, A.; Blaxter, M. Molecular barcodes for soil nematode identification. Mol. Ecol. 2002, 11, 839-850. [CrossRef] [PubMed]

12. Hebert, P.D.N.; Ratnasingham, S.; De Waard, J.R. Barcoding animal life: Cytochrome c oxidase subunit 1 divergences among closely related species. Proc. R. Soc. B Biol. Sci. 2003, 270, 96-99. [CrossRef] [PubMed]

13. Stoeckle, M. Taxonomy, DNA, and the Bar Code of Life. Bioscience 2003, 53, 796-797. [CrossRef]

14. Blouin, M.S.; Yowell, C.A.; Courtney, C.H.; Dame, J.B. Substitution bias, rapid saturation, and the use of mtDNA for nematode systematics. Mol. Biol. Evol. 1998, 15, 1719-1727. [CrossRef]

15. DeRycke, S.; Remerie, T.; Vierstraete, A.; Backeljau, T.; Vanfleteren, J.; Vincx, M.; Moens, T. Mitochondrial DNA variation and cryptic speciation within the free-living marine nematode Pellioditis marina. Mar. Ecol. Prog. Ser. 2005, 300, 91-103. [CrossRef]

16. DeRycke, S.; Vanaverbeke, J.; Rigaux, A.; Backeljau, T.; Moens, T. Exploring the Use of Cytochrome Oxidase c Subunit 1 (COI) for DNA Barcoding of Free-Living Marine Nematodes. PLoS ONE 2010, 5, e13716. [CrossRef] 
17. Morise, H.; Miyazaki, E.; Yoshimitsu, S.; Eki, T. Profiling Nematode Communities in Unmanaged Flowerbed and Agricultural Field Soils in Japan by DNA Barcode Sequencing. PLoS ONE 2012, 7, e51785. [CrossRef]

18. Janssen, T.; Karssen, G.; Verhaeven, M.; Coyne, D.; Bert, W. Mitochondrial coding genome analysis of tropical root-knot nematodes (Meloidogyne) supports haplotype based diagnostics and reveals evidence of recent reticulate evolution. Sci. Rep. 2016, 6, 22591. [CrossRef]

19. Sánchez-Monge, A.; Janssen, T.; Fang, Y.; Couvreur, M.; Karssen, G.; Bert, W. mtCOI successfully diagnoses the four main plant-parasitic Aphelenchoides species (Nematoda: Aphelenchoididae) and supports a multiple origin of plant-parasitism in this paraphyletic genus. Eur. J. Plant Pathol. 2017, 148, 853-866. [CrossRef]

20. Janssen, T.; Karssen, G.; Orlando, V.; Subbotin, S.A.; Bert, W. Molecular characterization and species delimiting of plant-parasitic nematodes of the genus Pratylenchus from the penetrans group (Nematoda: Pratylenchidae). Mol. Phylogenet. Evol. 2017, 117, 30-48. [CrossRef]

21. Ahmed, M.; Back, M.A.; Prior, T.; Karssen, G.; Lawson, R.; Adams, I.; Sapp, M. Metabarcoding of soil nematodes: The importance of taxonomic coverage and availability of reference sequences in choosing suitable marker(s). Metabarcoding Metagenomics 2019, 3, 36408. [CrossRef]

22. Fonseca, V.G.; Carvalho, G.R.; Nichols, B.; Quince, C.; Johnson, H.F.; Neill, S.P.; Lambshead, J.D.; Thomas, W.K.; Power, D.; Creer, S. Metagenetic analysis of patterns of distribution and diversity of marine meiobenthic eukaryotes. Glob. Ecol. Biogeogr. 2014, 23, 1293-1302. [CrossRef]

23. Porazinska, D.L.; Giblin-Davis, R.M.; Faller, L.; Farmerie, W.; Kanzaki, N.; Morris, K.; Powers, T.O.; Tucker, A.E.; Sung, W.; Thomas, W.K. Evaluating high-throughput sequencing as a method for metagenomic analysis of nematode diversity. Mol. Ecol. Resour. 2009, 9, 1439-1450. [CrossRef] [PubMed]

24. Porazinska, D.L.; Sung, W.; Giblin-Davis, R.M.; Thomas, W.K. Reproducibility of read numbers in high-throughput sequencing analysis of nematode community composition and structure. Mol. Ecol. Resour. 2009, 10, 666-676. [CrossRef] [PubMed]

25. Macheriotou, L.; Guilini, K.; Bezerra, T.N.; Tytgat, B.; Nguyen, D.T.; Nguyen, T.X.P.; Noppe, F.; Armenteros, M.; Boufahja, F.; Rigaux, A.; et al. Metabarcoding free-living marine nematodes using curated 18S and CO1 reference sequence databases for species-level taxonomic assignments. Ecol. Evol. 2019, 9, 1211-1226. [CrossRef]

26. Schenk, J.; Kleinbölting, N.; Traunspurger, W. Comparison of morphological, DNA barcoding, and metabarcoding characterizations of freshwater nematode communities. Ecol. Evol. 2020, 10, 2885-2899. [CrossRef]

27. Waeyenberge, L.; De Sutter, N.; Viaene, N.; Haegeman, A. New Insights into Nematode DNA-metabarcoding as Revealed by the Characterization of Artificial and Spiked Nematode Communities. Diversity 2019, 11, 52. [CrossRef]

28. Griffiths, B.; De Groot, G.; Laros, I.; Stone, D.; Geisen, S. The need for standardisation: Exemplified by a description of the diversity, community structure and ecological indices of soil nematodes. Ecol. Indic. 2018, 87, 43-46. [CrossRef]

29. Geisen, S.; Snoek, L.B.; Hooven, F.C.T.; Duyts, H.; Kostenko, O.; Bloem, J.; Martens, H.; Quist, C.W.; Helder, J.A.; Van Der Putten, W.H. Integrating quantitative morphological and qualitative molecular methods to analyse soil nematode community responses to plant range expansion. Methods Ecol. Evol. 2018, 9, 1366-1378. [CrossRef]

30. Kebschull, J.M.; Zador, A.M. Sources of PCR-induced distortions in high-throughput sequencing data sets. Nucleic Acids Res. 2015, 43, 143. [CrossRef]

31. Darby, B.; Todd, T.C.; Herman, M.A. High-throughput amplicon sequencing of rRNA genes requires a copy number correction to accurately reflect the effects of management practices on soil nematode community structure. Mol. Ecol. 2013, 22, 5456-5471. [CrossRef] [PubMed]

32. Holovachov, O.; Haenel, Q.; Bourlat, S.J.; Jondelius, U. Taxonomy assignment approach determines the efficiency of identification of OTUs in marine nematodes. R. Soc. Open Sci. 2017, 4, 170315. [CrossRef] [PubMed]

33. Armenteros, M.; Rojas-Corzo, A.; Ruiz-Abierno, A.; DeRycke, S.; Backeljau, T.; Decraemer, W. Systematics and DNA barcoding of free-living marine nematodes with emphasis on tropical desmodorids using nuclear SSU rDNA and mitochondrial COI sequences. Nematology 2014, 16, 979-989. [CrossRef] 
34. Prosser, S.W.J.; Velarde-Aguilar, M.G.; León-Règagnon, V.; Hebert, P.D.N. Advancing nematode barcoding: A primer cocktail for the cytochrome c oxidase subunit I gene from vertebrate parasitic nematodes. Mol. Ecol. Resour. 2013, 13, 1108-1115. [CrossRef]

35. Sapkota, R.; Nicolaisen, M. High-throughput sequencing of nematode communities from total soil DNA extractions. BMC Ecol. 2015, 15, 3. [CrossRef]

36. Hadziavdic, K.; Lekang, K.; Lanzen, A.; Jonassen, I.; Thompson, E.M.; Troedsson, C. Characterization of the 18S rRNA Gene for Designing Universal Eukaryote Specific Primers. PLoS ONE 2014, 9, 87624. [CrossRef]

37. Avó, A.P.; Daniell, T.J.; Neilson, R.; Oliveira, S.; Branco, J.; Adão, H. DNA Barcoding and Morphological Identification of Benthic Nematodes Assemblages of Estuarine Intertidal Sediments: Advances in Molecular Tools for Biodiversity Assessment. Front. Mar. Sci. 2017, 4, 1-16. [CrossRef]

38. DeRycke, S.; Backeljau, T.; Vlaeminck, C.; Vierstraete, A.; Vanfleteren, J.; Vincx, M.; Moens, T. Spatiotemporal analysis of population genetic structure in Geomonhystera disjuncta (Nematoda, Monhysteridae) reveals high levels of molecular diversity. Mar. Biol. 2007, 151, 1799-1812. [CrossRef]

39. Lazarova, S.S.; Malloch, G.; Oliveira, C.M.; Hübschen, J.; Neilson, R. Ribosomal and Mitochondrial DNA Analyses of Xiphinema americanum-Group Populations. J. Nematol. 2006, 38, 404-410.

40. Sapkota, R.; Nicolaisen, M. Cropping history shapes fungal, oomycete and nematode communities in arable soils and affects cavity spot in carrot. Agric. Ecosyst. Environ. 2018, 257, 120-131. [CrossRef]

41. Blaxter, M.; De Ley, P.; Garey, J.R.; Liu, L.X.; Scheldeman, P.; Vierstraete, A.; Vanfleteren, J.R.; Mackey, L.Y.; Dorris, M.; Frisse, L.M.; et al. A molecular evolutionary framework for the phylum Nematoda. Nat. Cell Biol. 1998, 392, 71-75. [CrossRef] [PubMed]

42. Kudjordjie, E.N.; Sapkota, R.; Steffensen, S.K.; Fomsgaard, I.S.; Nicolaisen, M. Maize synthesized benzoxazinoids affect the host associated microbiome. Microbiome 2019, 7, 59. [CrossRef] [PubMed]

43. Rognes, T.; Flouri, T.; Nichols, B.; Quince, C.; Mahé, F. VSEARCH: A versatile open source tool for metagenomics. PeerJ 2016, 4, 2584. [CrossRef] [PubMed]

44. Quast, C.; Pruesse, E.; Yilmaz, P.; Gerken, J.; Schweer, T.; Yarza, P.; Peplies, J.; Glöckner, F.O. The SILVA ribosomal RNA gene database project: Improved data processing and web-based tools. Nucleic Acids Res. 2012, 41, D590-D596. [CrossRef] [PubMed]

45. Yilmaz, P.; Parfrey, L.W.; Yarza, P.; Gerken, J.; Pruesse, E.; Quast, C.; Schweer, T.; Peplies, J.; Ludwig, W.; Glöckner, F.O. The SILVA and “All-species Living Tree Project (LTP)” taxonomic frameworks. Nucleic Acids Res. 2013, 42, 643-648. [CrossRef] [PubMed]

46. Caporaso, J.G.; Kuczynski, J.; Stombaugh, J.; Bittinger, K.; Bushman, F.D.; Costello, E.K.; Fierer, N.; Peña, A.G.; Goodrich, J.K.; Gordon, J.I.; et al. QIIME allows analysis of high-throughput community sequencing data. Nat. Methods 2010, 7, 335-336. [CrossRef] [PubMed]

47. McMurdie, P.J.; Holmes, S. phyloseq: An R Package for Reproducible Interactive Analysis and Graphics of Microbiome Census Data. PLoS ONE 2013, 8, 61217. [CrossRef]

48. Treonis, A.M.; Unangst, S.K.; Kepler, R.M.; Buyer, J.S.; Cavigelli, M.A.; Mirsky, S.B.; Maul, J.E. Characterization of soil nematode communities in three cropping systems through morphological and DNA metabarcoding approaches. Sci. Rep. 2018, 8, 2004. [CrossRef]

49. Deagle, B.E.; Jarman, S.N.; Coissac, E.; Pompanon, F.; Taberlet, P. DNA metabarcoding and the cytochrome c oxidase subunit I marker: Not a perfect match. Biol. Lett. 2014, 10, 20140562. [CrossRef]

50. Bik, H.; Sung, W.; De Ley, P.; Baldwin, J.G.; Sharma, J.; Rocha-Olivares, A.; Thomas, W.K. Metagenetic community analysis of microbial eukaryotes illuminates biogeographic patterns in deep-sea and shallow water sediments. Mol. Ecol. 2011, 21, 1048-1059. [CrossRef]

51. Capra, E.; Giannico, R.; Montagna, M.; Turri, F.; Cremonesi, P.; Strozzi, F.; Leone, P.; Gandini, G.; Pizzi, F. A new primer set for DNA metabarcoding of soil Metazoa. Eur. J. Soil Biol. 2016, 77, 53-59. [CrossRef]

52. Creer, S.; Fonseca, V.G.; Porazinska, D.L.; Giblin-Davis, R.M.; Sung, W.; Power, D.; Packer, M.; Carvalho, G.R.; Blaxter, M.; Lambshead, P.J.D.; et al. Ultrasequencing of the meiofaunal biosphere: Practice, pitfalls and promises. Mol. Ecol. 2010, 19, 4-20. [CrossRef] [PubMed]

(C) 2020 by the authors. Licensee MDPI, Basel, Switzerland. This article is an open access article distributed under the terms and conditions of the Creative Commons Attribution (CC BY) license (http://creativecommons.org/licenses/by/4.0/). 